\title{
Biochemical Epidemiology
}

National Cancer Institute

\section{Source}

National Cancer Institute. Biochemical Epidemiology. NCI Thesaurus. Code C18644.

Biochemical epidemiology incorporates the collection of biologic specimens in epidemiologic investigations, which can be analyzed by laboratory techniques to gain better insights into specific environmental and genetic determinants of disease, precursor states, and the pathogenic mechanisms. 